\title{
Dice Similarity Based Gaussian Deep Recurrent Neural Learning for Classification and Prediction with Big Data Analytics
}

\author{
S Arun Kumar, M Venkatesulu
}

\begin{abstract}
Big data analytics is a process of gathering large volume of data and organizing the present and past events to predict future events. Analyzing such a huge volume of data is not a simple task. Therefore, processing large data is a challenging one to predict an accurate event. The conventional techniques handling the large volume of data but the accurate prediction was not obtained since it failed to progressively learn the higher level features from raw inputs. An efficient Dice similarity based Gaussian Deep Recurrent Neural Learning Classifier (DS-GDRNLC) model is developed to enhance the prediction performance in terms of prediction time, prediction accuracy with big data. Initially, DS-GDRNLC model gathers huge volume of data from the big dataset (DS). After that, the gathered data are trained with several layers such as input layer, two hidden layers and output layer. The numbers of data are given to the input layer for performing the classification. Then the proposed DS-GDRNLC model uses two hidden layers to repeatedly learn the input data using a regression function. The regression function uses the dice similarity coefficient to find the relationship between the data and the predicted class. Then the analyzed results at the hidden layers are fed into the output layer. The Gaussian activation function is used at the output layer to verify the similarity value and mean of class. If the similarity value is closer to the mean of class, then the data are classified into that specific class. In this way, all the input data are accurately classified into the different classes resulting improves the Prediction Accuracy (PA). Finally, the training error rate is calculated for each classification results for obtaining the higher PA. This process repeated until the minimum error is obtained. Experimental evaluation is performed with big DS using different metrics such as PA, precision, recall, F-measure and Prediction Time (PT). The observed results confirm that the DS-GDRNLC model efficiently increases the $P A$, precision, recall as well as $F$-measure and minimizes the PT than the state-of-the-art methods.
\end{abstract}

Keywords: Predictive analytics, big data, Gaussian Deep Recurrent Neural Learning Classifier, regression, dice similarity coefficient, Gaussian activation function

\section{INTRODUCTION}

$\mathrm{W}$ statistics that extracting more relevant information from the large volume of data and predict the future and behavior patterns. In recent days, big data is the fastest and more

Revised Manuscript Received on July 22, 2019.

*S Arun Kumar

S Arun Kumar*, Department of Computer Science and Enginnering, Kalasalingam Academy of Research and Education, Krishnankoil, India. Email: arunsphd@gmail.com

M Venkatesulu, Department of Information Technology, Kalasalingam Academy of Research and Education, Krishnankoil, India.

Email: m.venkatesulu@klu.ac.in broadly used in several application fields such as, healthcare, weather, agriculture, business, marketing, banking and so on. Recently, several research works have been developed for predictive analytics with big data. However, it faces several challenges for accurate prediction.

The support vector trained multilayer neural network (SVM- trained MNN) was developed in [1] for classifying the big data with the help of map-reduce function. The method minimizes the PT but the accurate classification was not achieved. In [2], a CNN-based multimodal disease risk prediction (CNN-MDRP) algorithm was presented with big data. The designed algorithm increased the PA but it has higher time to predict the disease.

A statistical assessment model of the healthcare information system was presented in [3] for predictive analysis with big data. Though the accuracy and F-measure were improved, the time consumption was not minimized using statistical assessment model. Artificial Intelligence (AI) methods were developed in [4] to predict disease with Big Data. But the methods failed to perform accurate disease prediction. For predicting malaria disease, an artificial neural network (ANN) was introduced in [5]. But the error rate of the prediction was not minimized. A deep learning paradigm was developed in [6] for increasing the health prediction using big data. But the repeated data learning was not performed to achieve higher accuracy.

In [7], an ensemble multi-label classification technique was designed to identify the disease risk at an earlier stage. But the designed technique failed to minimize the error. A spark based machine learning technique was developed in [8] for remote health status prediction. The designed technique failed to predict a variety of diseases.

A machine-learning-based diagnosis scheme was introduced in [9] to predict heart disease with minimum computation time. But the performance of disease prediction was not improved while considering the large volume of data. A decision tree classification technique was developed in [10] to improve the performance of heart disease prediction. But, the false positive rate (FPR) was not minimized.

Abovementioned issues are overcome by presenting a novel DS-GDRNLC model.The major contribution of the proposed DS-GDRNLC model is summarized as follows

$>$ To improve the PA, DS-GDRNLC model is introduced by repeatedly learning the input data in the hidden layers. In the hidden layers, the input data are analyzed using the regression function. To find the relationship between dependent variable (i.e. data) and independent data (i.e. predicted class), the regression function uses the dice similarity coefficient.Based on the similarity values, the data are classified at the

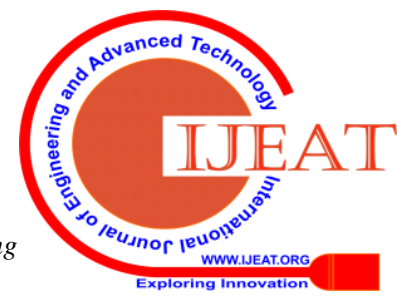




\section{Dice Similarity Based Gaussian Deep Recurrent Neural Learning For Classification And Prediction With Big Data Analytics}

output layer. This helps to improve the precision, recall and F-measure.

$>$ To minimize the PT, the Gaussian activation function is used at the output layer for analyzing the similarity value and mean value of the predictive class. The similarity value which is closer to the mean is classified into the particular class.

The rest of the paper is structured into five different sections. Section 2 reveals the related works. In Section 3, the proposed DS-GDRNLC model is explained with the architecture diagram. In Section 4, experimental evaluation is performed with big data. The experimental results are discussed with different parameters in Section 5. Section 6 provides the conclusion of the paper.

\section{RELATED WORKS}

Machine learning methods with rule-based classification were introduced in [11] for forecasting a variety of liver diseases. But the accurate prediction was not achieved with minimum time. A deep neural network (DNN) and long-short-term memory (LSTM) learning models were developed in [12] for predicting various infectious diseases. But the prediction performance was not increased.

A big data analytics-enabled transformation method was introduced in [13] and applied to the healthcare organization for predicting the disease. The performance of accuracy and time complexity remained unsolved. An efficient and privacy-preserving disease risk prediction (EPDP) system was developed in [14] for disease risk prediction. The system failed to improve the accuracy of diagnosis results at an earlier stage.

A probabilistic data acquisition technique was designed in [15] to predict the future health status of the patients. Though the designed technique improves the PA, the PT was not minimized. The three machine learning classification techniques were developed in [16] to predict diabetes at an early stage. The algorithms failed to predict or diagnose other diseases.

A distributed association rule-based classification method was developed in [17] based on MapReduce for big data analytics. The method improves the accuracy but the error rate was not minimized. A predictive analysis algorithm using Hadoop/Map Reduce was developed in [18] to forecast different types of diabetes with high accuracy. But the PT was not minimized.

Predictive analytics with big data in emergency care was developed [19] to predict the disease. But the performance of the disease prediction remained unsolved. A recurrent convolutional neural network (RCNN) was developed in [20] to evaluate the disease risk. The disease risk prediction time was not minimized.

Major issues in big data predictive analysis are less prediction accuracy, high prediction time and error rate. These existing issues are overcome by presenting a novel DS-GDRNLC model. The brief explanation of DS-GDRNLC model is presented in the next section.

\section{METHODOLOGY}

The DS-GDRNLC Model is developed for predicting future events with a large volume of data. The management and analysis of big data are the most significant emerging needs as the huge volume increasing the complexity of data being created or collected. Therefore, deep recurrent neural learning is introduced to effectively perform predictive analytics. The "deep learning" in the proposed DS-GDRNLC model refers to use the number of hidden layers for deeply analyzing the input data through which the data is transformed to obtain better outcomes.

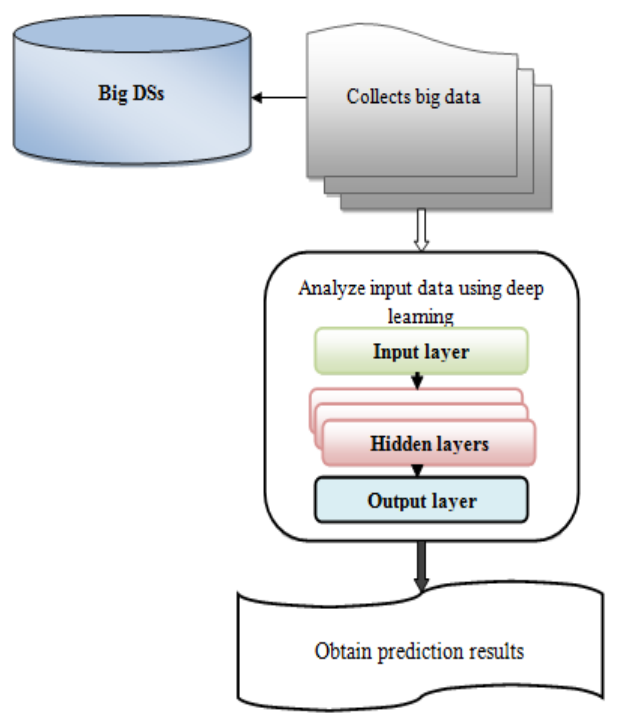

Figure 1 architecture of DS-GDRNLC model

More precisely, deep learning is the sequence of transformations from the input to output. The normal neural learning uses the one input layer, one hidden and one output layer for processing the input big data. But the deep learning includes one input layer, two or more hidden layers and one output layer. The different hidden layers are used to deeply learn the input data and provide accurate prediction results at the output layer by using regression function. A function of the independent variables called the regression function is to be estimated. Regression fuction focus is on the relationship between a dependent variable (i.e. Class) and one or more independent variables (i.e. data') Regression Fuction helps one understand how the typical value of the dependent variable (i.e. Class) changes when any one of the independent variables is varied, while the other independent variables are held fixed. The processing diagram of the proposed DS-GDRNLC model is shown in figure 1.

Figure 1 depicts the architecture of the proposed DS-GDRNLC model to obtain better prediction results with minimum time complexity. Initially, the big DS is considered for predictive analytics. The number of data is taken from the big DS. Using deep recurrent neural learning, the data analysis is performed with the input data. The input data is fed into the input layer of the deep architecture. Then the incoming data are analyzed in the hidden layers. Finally, the results are obtained at the output layer. The structural diagram of the deep recurrent neural leering technique is shown in figure 2 . 


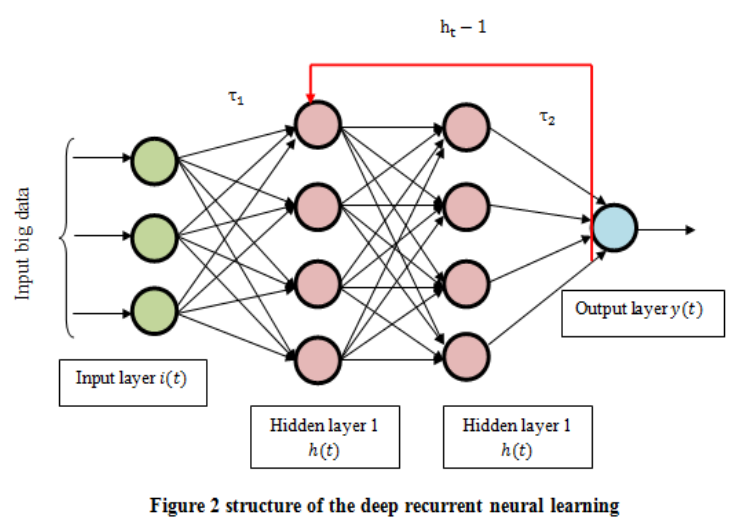

Figure 2 illustrates the structure of the deep recurrent neural learning with the one hidden layer, two hidden layers and output layer. The numbers of big data are given to the input $i(t)$ layer $\mathrm{d}_{1}, \mathrm{~d}_{2}, \mathrm{~d}_{3}, \ldots . \mathrm{d}_{\mathrm{n}}$ at time' $\mathrm{t}$ '. The deep recurrent neural learning includes the neurons-like the nodes into different layers and interconnected directly into the next successive layers with adjustable weights $\tau_{1}, \tau_{2}$. The nodes in the one layer are fully connected to the consequent layers and repetitively perform the deep learning of input data hence the name is called as Recurrent Deep neural network. The inputs are transformed into the hidden layers. The adjustable weights between the input and hidden layer is represented as $\tau_{1}$ as shown in figure 2 . The input layer of the deep learning is expressed as,

$$
i(t)=\sum_{i=1}^{n} \mathrm{~d}_{\mathrm{i}}(\mathrm{t}) * \tau_{1}
$$

In $(1), i(t)$ represents the input, $\mathrm{d}_{\mathrm{i}}(\mathrm{t})$ denotes a big data at a time' $t$ ', $\tau_{1}$ denotes an adjustable weights between the input and hidden layer. In the proposed deep learning, there are two hidden layers are used for predicting the future results with big data. The regression is used in hidden layers to analysis the input data. The similarity between dependent variable (i.e. input data) and one or more independent variables are evaluated by the regression analysis. The variable whose values to be predicted is known as dependent variable and independent variable is number of input data. The relationship is measured based on the similarity function. The dice similarity is employed for finding relationship between dependent variable and independent variable which is computed as follows,

$$
\beta=2 * \frac{d_{i} \cap c_{i}}{\left|d_{i}\right|+\left|c_{i}\right|}(2)
$$

In (2), $\beta$ represent the regression coefficient, $d_{i}$ represents the independent variable (i.e. data), $c_{i}$ denotes a predicted dependent variable (i.e. class). $\left|d_{i}\right|$ and $\left|c_{i}\right|$ represents the cardinalities of the two sets (i.e. a number of a elements in each set). The dice similarity is equals to the ratio of the mutual independence between the two variables and the sum of the number of elements in each set. The dice similarity coefficient $(\beta)$ provides the similarity value between 0 and 1 . Based on the regression analysis, the output of the hidden layer is represented as ' $h(t)$ '.

$$
h(t)=\tau_{1} * i(t)+\tau_{h} * h(t-1)
$$

In (3) $h(t)$ denotes a hidden layer output at a time ' $t$ '. Here, ' $h(t-1)$ ' represents a unit time delay output from hidden layer 2 to hidden layer 1 and ' $\tau_{h}$ ' denotes a weights of the hidden layer, $\tau_{1}$ represents a weight between input and hidden layers, $i(t)$ represents the input. The output of the second hidden layer is feedback into the input of the first hidden layer and repeatedly learning the input data and given to the output layer.

Then the similarity values are transformed into the output layer where the similarity values are verified and predict the results. The hidden layer output is transformed into the output layer.

$$
y(t)=\gamma_{f}\left(\tau_{2} * h(t)\right)(4)
$$

In (4), $y(t)$ represents the output of the deep recurrent neural learning, $\tau_{2}$ represents the adjustable weights between the hidden layer and output layer, $h(t)$ denotes an output of the hidden layer. $\gamma_{f}$ denotes a activation. The proposed DS-GDRNLC model uses the Gaussian activation function to classify the data into a particular class uses the mean and deviation.

$$
\gamma_{f}=e^{\left(-\frac{(\beta-\mu)^{2}}{2 \sigma^{2}}\right)}
$$

In (5), $\beta$ denotes a similarity value, $\mu$ denotes a mean of the class, ' $\sigma$ ' represents the deviation from the mean. If the similarity value is closer to the mean, the activation function returns ' 1 '. It means that the data are classified into the particular class. Otherwise, the similarity is deviated from the mean of a particular class and the activation function returns ' 0 '. In this way, all the data are correctly classified into the particular class. After the classification, the error rate is computed for obtaining the higher PA. The error rate is calculated based on the difference between the squared difference between the actual and predicted results. It is mathematically formulated as follows,

$$
E=\left\{y_{a}(t)-y(t)\right\}^{2}
$$

In (6), $E$ denotes an error, $y_{a}(t)$ denotes an actual output, $y(t)$ represents the predicted result. Based on the error rate, the weights of the connection between the layers are adjusted and the process is repeated until it reaches the minimum training error.

$$
T_{e}=\arg \min E(7)
$$

In (7), $T_{e}$ represents the minimum training error, arg $\min$ denotes an argument of the minimum function, $E$ represents the error. This process minimizes the incorrect prediction of the future events and enhances the prediction accuracy. The algorithmic process of the DS-GDRNLC model is described as follows, 


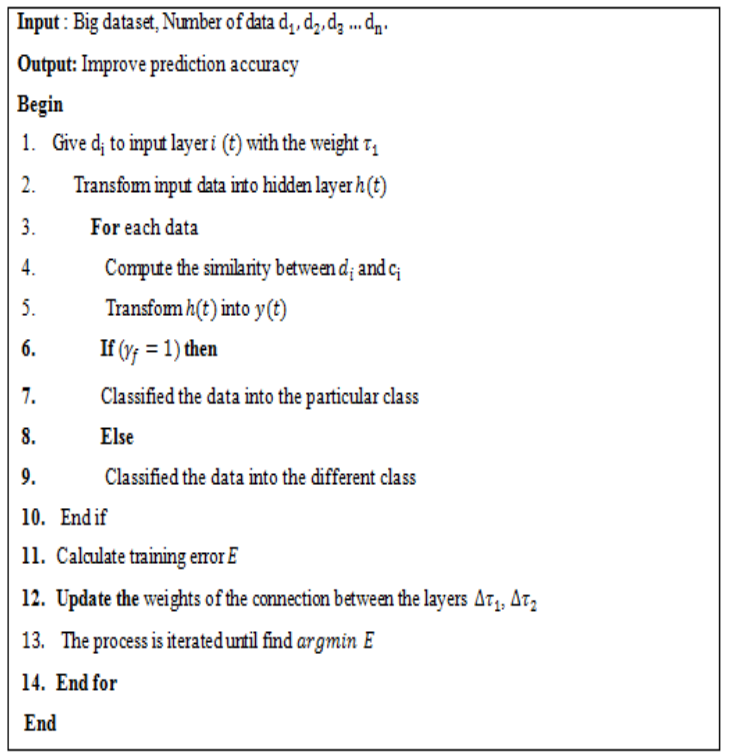

Algorithm 1 Dice similarity based Gaussian deep recurrent neural learning
The Pima Indians Diabetes Database is used to predict the patient affected by diabetes or not, based on certain analytical measurements information's. In this DS, all the patients here are females with 21 years old of Pima Indian heritage. The DS comprises the 9 attributes such as pregnancies, Glucose, blood pressure, skin thickness, insulin, BMI, diabetes pedigree function, age, and outcome.

The experimental evaluation is done with the above said medical DSs for predicting the two diseases with the patient information's. The different parameters such as PA, precision, recall, F-measure and $\mathrm{PT}$ are evaluated with number of patient files.

\section{RESULTS AND DISCUSSION}

In this section, Results and discussion of DS-GDRNLC and existing methods namely SVM-trained multilayer neural network (MNN) [1], CNN-MDRP [2], RCNN [3] are described. Various metrics namely PA, precision, recall, F-measure and PT with number of files are presented to validate the DS-GDRNLC technique in healthcare industry.

\section{A. Performance analysis of prediction accuracy}

PA is measured as ratio of number of patient files are correctly predicted as disease or not to total number of input files taken for the experimental. PA is formalized as follows, prediction accuracy $=$

$\frac{\text { Number of files are correctly predicted as disease or not }}{n} * 100$

In (8), $n$ denotes a number of the patient files. The PA is measured in terms of percentage $(\%)$.

PA of three different methods is computed with number of patient files. While considering 20 patient files, the DS-GDRNLC technique correctly predicts 17 patient files as disease or not and PA is $85 \%$. The PA of the other two methods is $75 \%$ and $70 \%$ respectively. Followed by, the nine runs are carried out with a number of patients' files. The PA of the proposed DS-GDRNLC technique is improved when compared to existing SVM-trained MNN [1] , CNN-MDRP [2] and RCNN [3] respectively. The ten different results are shown in figure 3 .

Table 1. Tabulation for prediction accuracy made with existing methods SVM-trained MNN [1], CNN-MDRP [2], RCNN (recurrent convolution neural network) [3] are implemented in Java Language to predict the diseases at an earlier stage. Big data (i.e. patient's information) are gathered from Cardiovascular Disease DS (https://www.kaggle.com/sulianova/cardiovascular-disease-

DS) and Pima Indians Diabetes Database (https://www.kaggle.com/uciml/pima-indians-diabetes-datab ase).

Cardiovascular Disease DS comprises 70,000 records of patient's data with 13 attributes. This DS is used to identify the conditions that involve blocked blood vessels that direct to a heart attack, chest pain (angina) or stroke. The attributes are id, age, gender, height, weight, ap_hi (systolic blood pressure), ap_lo (diastolic blood pressure), cholesterol, glucose, smoke, alco, active and cardio.

\begin{tabular}{|c|c|c|c|c|}
\hline \multirow{2}{*}{$\begin{array}{c}\text { Number } \\
\text { of } \\
\text { patient } \\
\text { files }\end{array}$} & DS-GDRNLC & $\begin{array}{c}\text { PVM-trained } \\
\text { MNN }\end{array}$ & CNN-MDRP & RCNN \\
\cline { 2 - 5 } & & 75 & 70 & 50 \\
\hline $\mathbf{2 0}$ & 85 & 80 & 73 & 55 \\
\hline $\mathbf{4 0}$ & 90 & 83 & 72 & 57 \\
\hline $\mathbf{6 0}$ & 92 & 80 & 70 & 61 \\
\hline $\mathbf{8 0}$ & 91 & 82 & 76 & 63 \\
\hline $\mathbf{1 0 0}$ & 94 & 83 & 75 & 65 \\
\hline $\mathbf{1 2 0}$ & 92 & 86 & 75 & 68 \\
\hline $\mathbf{1 4 0}$ & 91 & 81 & 75 & 70 \\
\hline $\mathbf{1 6 0}$ & 94 & 82 & 74 & 73 \\
\hline $\mathbf{1 8 0}$ & 91 & 84 & 80 & 75 \\
\hline $\mathbf{2 0 0}$ & 95 & & & \\
\hline
\end{tabular}




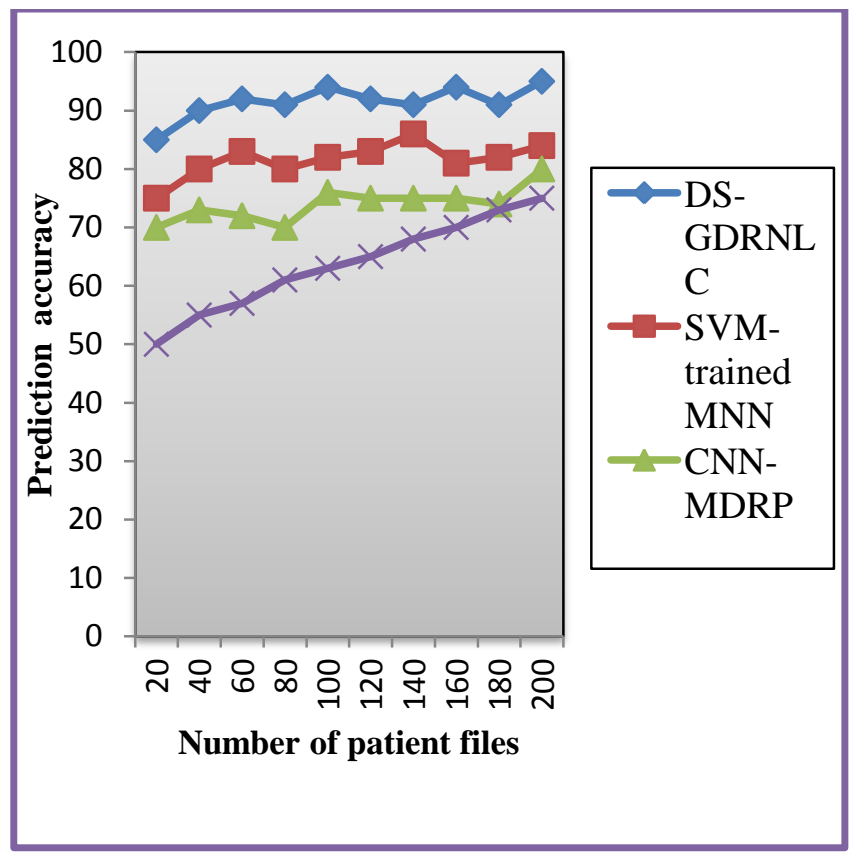

Figure 3 simulation results of number of patient files vs prediction accuracy

Figure 3 portrays results of PA versus number of patient files which is ranges from 20 to 200. Patient information's is gathered from big DS. The numbers of patient files is taken as input in ' $x$ ' direction and PA is obtained at ' $y$ ' direction. The above graph clearly shows that the DS-GDRNLC technique improves PA than the existing classification techniques. This improvement is achieved by applying a deep recurrent neural network to effectively learn the patient information's such as systolic blood pressure, diastolic blood pressure, cholesterol, glucose, insulin, BMI and so on. This patient information's are fed into the input layer. Then the input data are transformed into the hidden layers and repetitively learned using regression analysis and transferred to the output layer. The output layer uses the Gaussian activation function to correctly classify the patient files as cardiovascular disease and Diabetes disease. In this way, the diseases are correctly predicted with higher accuracy.

The ten different results of PA are compared with the existing classification techniques. Therefore, DS-GDRNLC technique enhances the PA by $12 \%, 24 \%$ and $45 \%$ as compared to existing [1], [2] and [3] respectively.

\section{B. Performance analysis of precision}

Precision is measured as number of patient files is accurately predicted as disease to the total number of input files taken for experimental evaluation. Precision is formulated as follows,

$$
\text { Precision }=\frac{T P}{T p+F P} * 100
$$

In (9), $T P$ denotes a true positive, $F P$ represents the false positive. True positive is numbers of files are correctly predicted as disease and FPR is numbers of files are incorrectly predicted as disease. Precision is calculated in percentage $(\%)$.
Table2. Tabulation form precision

\begin{tabular}{|c|c|c|c|c|}
\hline \multirow{2}{*}{$\begin{array}{c}\text { Number } \\
\text { of } \\
\text { patient } \\
\text { files }\end{array}$} & \multicolumn{4}{|c|}{ Precision (\%) } \\
\cline { 2 - 5 } & DS-GDRNLC & $\begin{array}{c}\text { SVM-trained } \\
\text { MNN }\end{array}$ & CNN-MDRP & RCNN \\
\hline $\mathbf{2 0}$ & 87 & 77 & 67 & 60 \\
\hline $\mathbf{4 0}$ & 94 & 86 & 74 & 63 \\
\hline $\mathbf{6 0}$ & 96 & 88 & 71 & 65 \\
\hline $\mathbf{8 0}$ & 95 & 87 & 78 & 67 \\
\hline $\mathbf{1 0 0}$ & 97 & 88 & 83 & 70 \\
\hline $\mathbf{1 2 0}$ & 95 & 89 & 84 & 73 \\
\hline $\mathbf{1 4 0}$ & 93 & 85 & 81 & 75 \\
\hline $\mathbf{1 6 0}$ & 96 & 88 & 80 & 77 \\
\hline $\mathbf{1 8 0}$ & 95 & 87 & 79 & 79 \\
\hline $\mathbf{2 0 0}$ & 97 & 89 & 85 & 82 \\
\hline
\end{tabular}

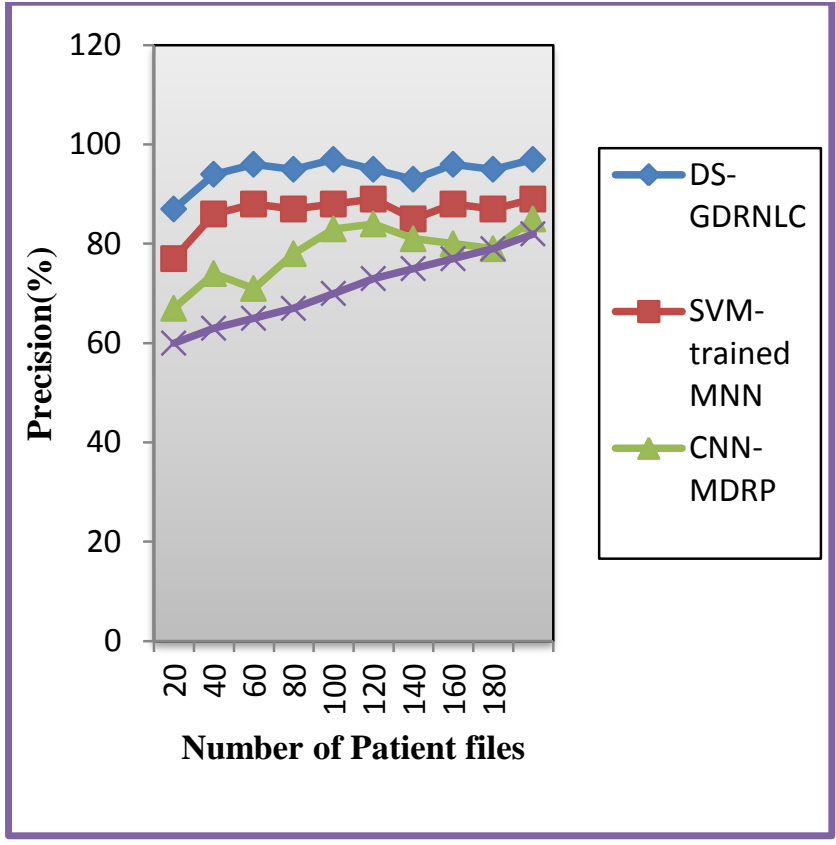

Figure4. Simulation results of number of patient file vs precision

Table 2 portrays the results of precision with number of patient files. Totally ten different results are described in table 1. For each iteration, the various precision results are obtained. The results show that the DS-GDRNLC technique improves precision when compared to the SVM-trained MNN [1], CNN-MDRP [2] and RCNN [3] respectively. The proposed DS-GDRNLC technique efficiently predicts cardiovascular disease and Diabetes disease at an earlier stage. By utilizing the deep learning classifier, the disease prediction is performed. Through the regression analysis, the incoming patient files are analyzed. The regression function analyzes the patient data and predicted class using the Dice similarity coefficient. Based on the similarity value, the Gaussian function classifies the disease types in an accurate manner. The error rate is calculated after the classification to reduce the FPR and enhances the true positive rate. 
The ten different precision results of DS-GDRNLC technique is compared with the precision of the conventional classification techniques. The comparison results clearly show that the precision is significantly increased by $9 \%, 21 \%$ and $33 \%$ than the existing SVM-trained MNN [1], CNN-MDRP [2] and RCNN [3] respectively.

\section{Performance analysis of Recall}

Recall is measured as ratio of the true positive to the sum of true positive and false negative results from the input files. The recall is formulated as follows,

$$
\text { Recall }=\frac{T P}{T p+F N}(10)
$$

In (10), $T P$ denotes a true positive, $F N$ represents the false negative. True positive is the numbers of files are correctly predicted and false negative is the numbers of files are incorrectly predicted as normal. Recall also measured in terms of percentage (\%).

The above table values are described that the evaluation results of recall using three different classification techniques namely DS-GDRNLC technique, SVM-trained MNN [1], CNN-MDRP [2] and RCNN [3]. For the experiential purposes, the number of patient's files taken as input in the range of 20 to 200 to compute the recall. The reported result confirms that the recall using proposed DS-GDRNLC technique is higher as compared to other classification techniques. The DS-GDRNLC technique performs deep learning with the patient data and predicts the disease affected by the patient at an earlier stage based on the similarity coefficient value. This in turn helps for increasing the patient files are correctly predicted at the output layer and minimizing the true negative rate. Therefore, the DS-GDRNLC technique improves the recall then the conventional technique. Because the conventional techniques failed to deeply analyze the patient data for accurate prediction.

Table 3. Tabulation for recall

\begin{tabular}{|c|c|c|c|c|}
\hline \multirow{2}{*}{$\begin{array}{c}\text { Number } \\
\text { of } \\
\text { patient } \\
\text { files }\end{array}$} & \multicolumn{5}{|c|}{ Recall (\%) } \\
\cline { 2 - 5 } & DS-GDRNLC & $\begin{array}{c}\text { SVM-trained } \\
\text { MNN }\end{array}$ & CNN-MDRP & RCNN \\
\hline $\mathbf{2 0}$ & 93 & 83 & 73 & 60 \\
\hline $\mathbf{4 0}$ & 94 & 86 & 83 & 62 \\
\hline $\mathbf{6 0}$ & 95 & 92 & 84 & 65 \\
\hline $\mathbf{8 0}$ & 96 & 91 & 83 & 67 \\
\hline $\mathbf{1 0 0}$ & 97 & 90 & 87 & 70 \\
\hline $\mathbf{1 2 0}$ & 96 & 91 & 86 & 72 \\
\hline $\mathbf{1 4 0}$ & 98 & 87 & 85 & 75 \\
\hline $\mathbf{1 6 0}$ & 97 & 90 & 84 & 77 \\
\hline $\mathbf{1 8 0}$ & 97 & 88 & 87 & 79 \\
\hline $\mathbf{2 0 0}$ & 97 & 92 & 88 & 81 \\
\hline
\end{tabular}

Let us consider the 20 patient files as input, true positive rate of DS-GDRNLC technique is 13 and the false negative rate is 1 , then the percentage of recall is $93 \%$. But the true positive rate and the false negative rate of SVM-trained MNN [1],
CNN-MDRP [2] and RCNN [3] are 10, 2 and 8, 3 respectively. Then the percentages of a recall are $83 \%$ and $73 \%$ respectively. Similarly, nine different results are obtained with a number of patients files. The ten different results of DS-GDRNLC technique is compared to the existing techniques. The results confirm that the recall is considerably increased by $8 \%, 15 \%$ and $36 \%$ then the state-of-the-art methods.

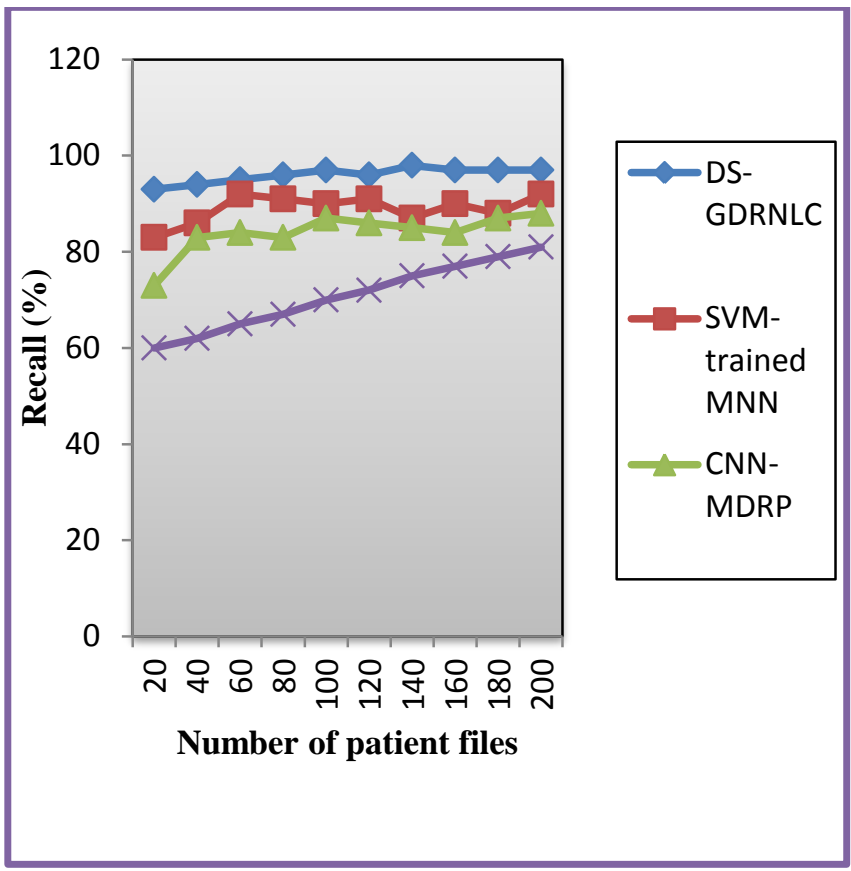

Figure 5. Simulation results of number of patients vs recall

\section{Performance analysis of F-measure}

F-measure is a measure of an evaluation accuracy of the classification algorithm and it is the average of the precision and recall. The F- measure is mathematically calculated as follows,

$$
F-\text { measure }=2 * \frac{P r * R c}{P r+R c} * 100
$$

From equation (11), $\operatorname{Pr}$ represents the precision, $R c$ denotes a recall. F- measure is measured in percentage (\%).

\begin{tabular}{|c|c|c|c|c|}
\hline \multirow[b]{2}{*}{$\begin{array}{l}\text { Num } \\
\text { ber of } \\
\text { patie } \\
\text { nt } \\
\text { files }\end{array}$} & \multicolumn{4}{|c|}{ F-Measure (\%) } \\
\hline & $\begin{array}{c}\text { DS-GDR } \\
\text { NLC }\end{array}$ & $\begin{array}{c}\text { SVM-trai } \\
\text { ned } \\
\text { MNN }\end{array}$ & $\begin{array}{c}\text { CNN-M } \\
\text { DRP }\end{array}$ & $\begin{array}{l}\text { RC } \\
\text { NN }\end{array}$ \\
\hline 20 & 90 & 80 & 70 & 63 \\
\hline 40 & 94 & 86 & 78 & 65 \\
\hline 60 & 95 & 90 & 85 & 67 \\
\hline 80 & 95 & 89 & 80 & 70 \\
\hline 100 & 97 & 89 & 85 & 72 \\
\hline 120 & 95 & 90 & 85 & 75 \\
\hline 140 & 95 & 86 & 83 & 78 \\
\hline 160 & 96 & 90 & 82 & 80 \\
\hline 180 & 96 & 87 & 83 & 82 \\
\hline
\end{tabular}

Table4. Tabulation for F-Measure 
Table 4 demonstrates the results of F-measure versus number of patient files ranges from of 20-200. For predicting the disease at an earlier stage, patient information's are gathered from DS and maintained in the file format. From the table value, it is proved that the proposed DS-GDRNLC model provides better F-measure results as compared to existing classification techniques. This is because of DS-GDRNLC model increases the precision as well as recall in the disease prediction analysis with big patient data. The F-measure is a measure of a test's accuracy of the DS-GDRNLC model which is obtained both precisions as well as recall.

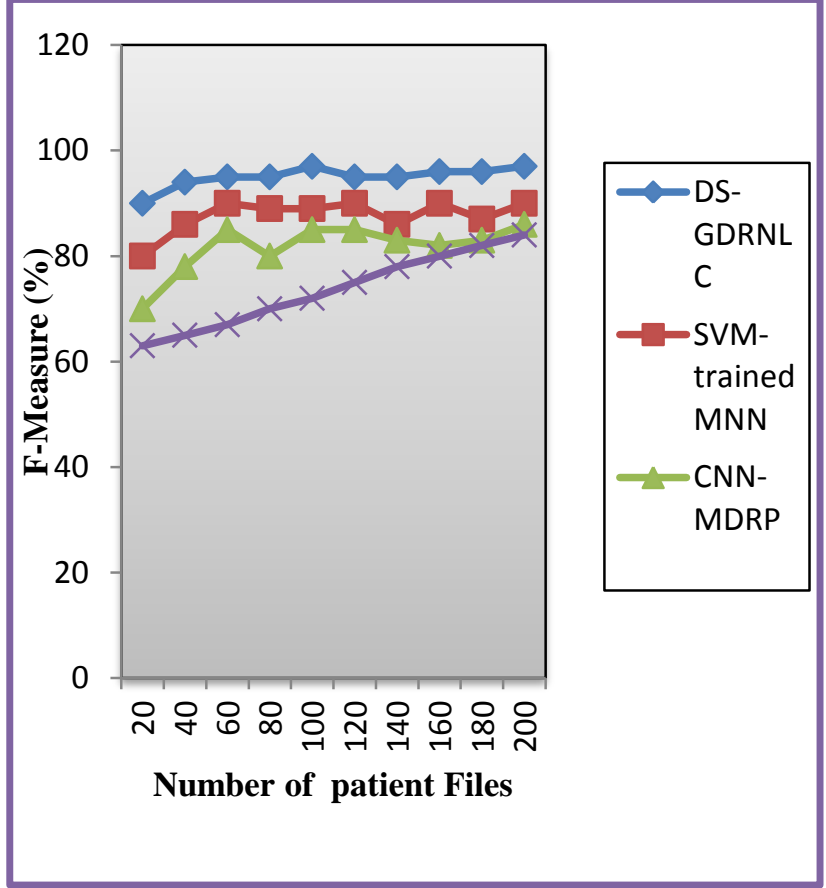

Figure 6 Simulation results of number of patient files vs

\section{F-Measure}

While considering the input count of the patient file is 20 , the precision and recall of DS-GDRNLC model are $87 \%$ and $93 \%$. Then the percentage of F-measure is $90 \%$. Similarly, the F-measure of the other two techniques is $80 \%$ and $70 \%$ respectively. Similarly, the patient files counts are increased for each run and the results are obtained. The performance results show that the DS-GDRNLC model outperforms well and the F-measure is improved by $8 \%, 17 \%$ and $30 \%$ than the SVM-trained MNN [1], CNN-MDRP [2] and RCNN [3] respectively. and $70 \%$ respectively. Similarly, the patient files counts are increased for each run and the results are obtained. The performance results show that the DS-GDRNLC model outperforms well and the F-measure is improved by $8 \%, 17 \%$ and $30 \%$ than the SVM-trained MNN [1], CNN-MDRP [2] and RCNN [3] respectively.

\section{E. Performance analysis of prediction time}

Prediction time is measured as amount of time taken to predict the disease type based on patient current information (i.e. file). PT is mathematically calculated as follows, prediction time $=n * T$ (predictingthedisease $)$ (12)

In (12), $n$ represents a number of files, $T$ denotes a time for predicting the disease. PT is measured in terms of milliseconds (ms).

Table 5. Tabulation for Precision Time

\begin{tabular}{|c|c|c|c|c|}
\hline $\begin{array}{l}\text { Number } \\
\text { of } \\
\text { patient } \\
\text { files }\end{array}$ & \multicolumn{5}{|l|}{ Precision time(\%) } & DS-GDRNLC & $\begin{array}{c}\text { SVM-trained } \\
\text { MNN }\end{array}$ & CNN-MDRP & RCNN \\
\hline $\mathbf{2 0}$ & 17 & 20 & 24 & 26 \\
\hline $\mathbf{4 0}$ & 22 & 24 & 28 & 29 \\
\hline $\mathbf{6 0}$ & 25 & 29 & 31 & 31 \\
\hline $\mathbf{8 0}$ & 30 & 34 & 37 & 38 \\
\hline $\mathbf{1 0 0}$ & 34 & 38 & 42 & 43 \\
\hline $\mathbf{1 2 0}$ & 37 & 43 & 47 & 49 \\
\hline $\mathbf{1 4 0}$ & 40 & 45 & 49 & 52 \\
\hline $\mathbf{1 6 0}$ & 44 & 48 & 51 & 54 \\
\hline $\mathbf{1 8 0}$ & 47 & 50 & 54 & 58 \\
\hline $\mathbf{2 0 0}$ & 50 & 54 & 58 & 60 \\
\hline
\end{tabular}

Figure 5 portrays the results of PT of proposed and existing methods with number of patient files. The above graphical result shows that the PT is said to be minimized when compared to the existing methods. By increasing the number of patient files, with the time taken to predict the disease also increases. But comparatively, the PT is found to be lesser usingDS-GDRNLC model due to the application of DS-GDRNLC. This classifier analyzes the input patient data at the two hidden layers and prediction results are obtained at the output layer. In that layer, the Gaussian activation function is used for analyzing the similarity value and mean value of the predictive class. If the similarity value is more close to the mean value, then the patient file is classified into the specific class. The classification results are used for predicting the disease with minimum time. The results of the PT of DS-GDRNLC model is minimized by $11 \%$ compared to the SVM-trained MNN [1] and $19 \%$ compared to CNN-MDRP [2] and $28 \%$ compared to RCNN [3].

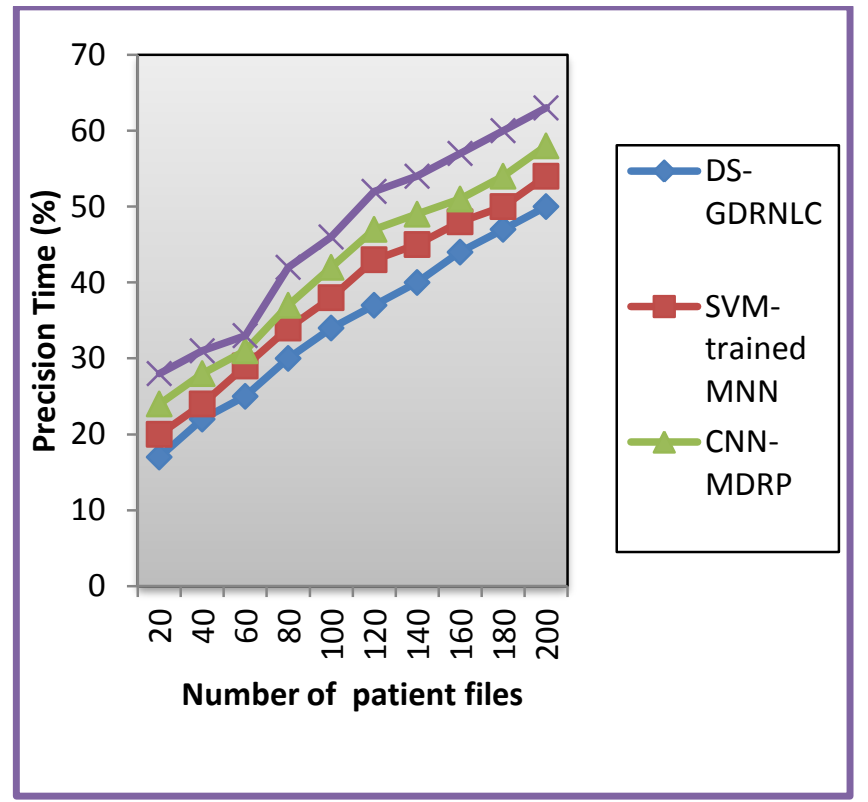

Figure 7 simulation results of number of patient files vs precision time

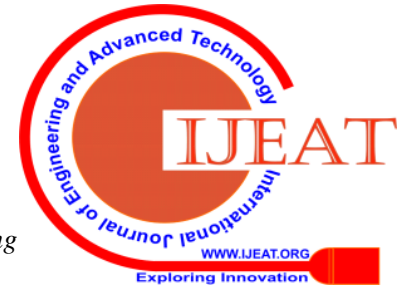




\section{Dice Similarity Based Gaussian Deep Recurrent Neural Learning For Classification And Prediction With Big Data Analytics}

The above discussion of the various performance metrics clearly shows that the DS-GDRNLC model effectively improves the disease prediction with higher accuracy and minimum time.

\section{CONCLUSION}

An efficient model called DS-GDRNLC is introduced to achieve higher PA and minimal time with the big data. The data are collected from the big DSs and given to the input layer of the recurrent deep neural learning classifier. Then the inputs are learned repetitively in two hidden layers using the regression analysis with dice similarity coefficient. The learned data are transferred to the output layer. In the output layer, the Gaussian activation function is used for classifying the data based on the similarity and mean value. Finally, the training error is calculated and the process is repeated until the minimum error is found. This helps to improve the accuracy and minimizes the FPR. Experimental evaluation is carried out using healthcare big DSs with the parameters such as PA, precision, recall, F-measure and PT. The observed result clearly shows that DS-GDRNLC model improves the disease PA, precision, recall, F-measure and minimizes the PT when compared to the state-of-the-art methods.

\section{ACKNOWLEDGMENT}

The first author is thankful to the management of Kalasalingam Academy of Research and Education for providing fellowship.

\section{REFERENCES}

1. Ahmad Ali AlZubi, "Big data analytic diabetics using map reduce and classification techniques", The Journal of Supercomputing, Springer, April 2018, pp 1-10

2. Min Chen, Yixue Hao, Kai Hwang, Lu Wang and Lin Wang, "Disease Prediction by Machine Learning over Big Data from Healthcare Communities", IEEE Access, Volume 5, April 2017, pp 8869 - 8879

3. C. B. Sivaparthipan, N. Karthikeyan and S. Karthik, "Designing statistical assessment healthcare information system for diabetics analysis using big data", Multimedia Tools and Applications, Springer, November 2018,pp 1-14

4. Zoie S.Y.Wong, Jiaqi Zhou, Qingpeng Zhang, "Artificial Intelligence for infectious disease Big Data Analytics", Infection, Disease \& Health, Elsevier, Volume 24, Issue 1, 2019,pp 44-48

5. Thakur Santosh and Dharavath Ramesh, "Artificial neural network based prediction of malaria abundances using big data: A knowledge capturing approach", Clinical Epidemiology and Global Health, Elsevier, Volume 7, 2019, pp 121-126

6. Hongye Zhong and Jitian Xiao, "Enhancing Health Risk Prediction with Deep Learning on Big Data and Revised Fusion Node Paradigm", Scientific Programming, Hindawi, Volume 2017, June 2017, pp1-18

7. Runzhi Li,Wei Liu, Yusong Lin, Hongling Zhao, and Chaoyang Zhang, "An Ensemble Multilabel Classification for Disease Risk Prediction", Journal of Healthcare Engineering, Hindawi, Volume 2017, June 2017, pp 1-10

8. Lekha R.Nair, Sujala D.Shetty, Siddhanth D.Shetty, “Applying spark based machine learning model on streaming big data for health status prediction", Computers \& Electrical Engineering, Elsevier, Volume 65, January 2018, pp 393-399

9. Amin Ul Haq, Jian Ping Li , Muhammad Hammad Memon, Shah Nazir , and Ruinan Sun, "A Hybrid Intelligent System Framework for the Prediction of Heart Disease Using Machine Learning Algorithms", Mobile Information Systems, Hindawi, Volume 2018, December 2018, pp 1-21

10. Jaymin Patel, Tejal Upadhyay, Dr. Samir Patel, "Heart Disease Prediction Using Machine learning and Data Mining Technique", International Journal of Computer Science \& Communication, Volume 7 , Issue 1,2015 , pp 129-137
11. Yugal Kumar and G. Sahoo, "Prediction of different types of liver diseases using rule based classification model", Technology and Health Care, Volume 21, Issue 5, 2013, pp 417-432

12. Sangwon Chae, Sungjun Kwon and Donghyun Lee, "Predicting Infectious Disease Using Deep Learning and Big Data", International Journal of Environmental Research and Public Health, Volume 15, 2018, pp 1-20

13. Yichuan Wang, LeeAnn Kung, William Yu Chung Wang, Casey G. Cegielski, "An integrated big data analytics-enabled transformation model: Application to health care", Information \& Management, Elsevier, Volume 55, Issue 1, 2018, pp 64-79

14. Xue Yang , Rongxing Lu, Jun Shao, Xiaohu Tang, Haomiao Yang, "An Efficient and Privacy-Preserving Disease Risk Prediction Scheme for E-Healthcare", : IEEE Internet of Things Journal, Volume 6, Issue 2, 2019, pp $3284-329$

15. Prasan Kumar Sahoo, Suvendu Kumar Mohapatra ,Shih-Lin Wu "Analyzing Healthcare Big Data With Prediction for Future Health Condition", IEEE Access, Volume 4, 2016, pp 9786 - 9799

16. Deepti Sisodia and Dilip Singh Sisodia, "Prediction of Diabetes using Classification Algorithm", Procedia Computer Science, Elsevier, Volume 132, 2018, pp 1578-1585

17. Alessio Bechini, Francesco Marcelloni, Armando Segatori, "A MapReduce solution for associative classification of big data", Information Sciences, Elsevier, Volume 332, 2016, pp 33-55

18. N.M. Saravanakumar, T.Eswari, P.Sampath, S.Lavanya, "Predictive Methodology for Diabetic Data Analysis in Big Data", Procedia Computer Science, Elsevier, Volume 50, 2015, pp 203-208

19. Alexander T.Janke BS, Daniel L.Overbeek MD, Keith E.Kocher MD MPH, Phillip D.Levy MD, MPH, "Exploring the Potential of Predictive Analytics and Big Data in Emergency Care", Annals of Emergency Medicine, Elsevier, Volume 67, Issue 2, 2016, pp 227-236

20. Mohd Usama, Belal Ahmad, Jiafu Wan, M. Shamim Hossain , Mohammed F. Alhamid, M. Anwar Hossain, "Deep Feature Learning for Disease Risk Assessment Based on Convolutional Neural Network With Intra-Layer Recurrent Connection by Using Hospital Big Data", IEEE Access, Volume 6, 2018, pp 67927 - 67939

\section{AUTHORS PROFILE}

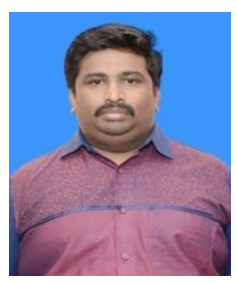

S. Arunkumar was born in Srivilliputtur, Tamil Nadu, and India. He received the B.E. degree in Computer Science and Engineering from the Anna University, Tamil Nadu, and India. In 2010, the M.Tech. Degree in Software Engineering from Karunya University, Coimbatore, Tamil Nadu, India. He has held Assistant Professor Positions at Kalaivani College of Technology, Coimbatore, Tamil Nadu, and India. And the University of Petroleum and Energy Studies, Dehradun, Uttarakhand. He is currently a Research Scholar at Kalasalingam Academy of Research and Education, Krishnankovil, Srivilliputtur, Tamil Nadu, and India. His research interests include Big Data Analytics, Distributed Computing, and Machine Learning.

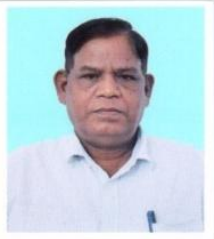

M.Venkatesulu received the postgraduate degree in Mathematics from Sri Venkateswara University, Tirupati, India, in 1975, and the Ph.d degree in mathematics from Indian Institute of Technology, Kanpur, India, in 1979. He worked as a faculty member at Shri Sathya Sa University, Prashanthinilayam, India Between 1983 and 2003. He also worked as a consultant for Satyam Computers, Hyderabad, India, for short period. He was Visiting Professor at University of Missouri, Kansas City, between August 2006 and May 2007. Currently he is working as a Senior Professor and Head of the Department of Information Technology at Kalasalingam Academy of Research and Education, Krishnankovil, Srivilliputtur, Tamil Nadu, and India. His area of interest includes differential equation, Image Processing, Cryptography, Bioinformatics, Big Data Analytics and Distributed Computing. 